\title{
Effect of Low-intensity Pulsed Ultrasound on Healing of Bone Defects in Rat Tibia as Measured by Reconstructed Three-dimensional Analysis of Micro CT Images
}

\author{
KEI WADA ${ }^{1}$, MUNEMITSU KAWANO ${ }^{1}$, YUICHI HEMMI ${ }^{1}$, REIJI SUZUKI ${ }^{2}$, KATSUNORI KUNOKI ${ }^{1}$, \\ HIROSHI SAKAGAMI ${ }^{3}$, HIROSHI KAWAZU ${ }^{4}$ and SATOSHI YOKOSE ${ }^{1}$ \\ ${ }^{1}$ Division of Endodontics and Operative Dentistry, Department of Restorative and Biomaterials Sciences, \\ Meikai University School of Dentistry, Saitama, Japan; \\ ${ }^{2}$ Division of Oral Rehabilitation, Department of Restorative and Biomaterials Sciences, \\ Meikai University School of Dentistry, Saitama, Japan; \\ ${ }^{3}$ Meikai University Research Institute of Odontology (M-RIO), Saitama, Japan; \\ ${ }^{4}$ Section of Continuous Education, School of Dentistry, Meikai University, Saitama, Japan
}

\begin{abstract}
Background/Aim: The aim of this study was to evaluate the effect of low-intensity pulsed ultrasound (LIPUS) on bone metabolism during the healing period in rat tibiae bone defects using micro-computed tomography (micro CT) imaging for three-dimensional morphological evaluation. Materials and Methods: The right tibia received ultrasound exposure (US group) every day, whereas the opposite side served as a control (Control group). At 1, 2, and 3 weeks after the operation, micro CT was performed, and the volume and surface area of new bone formation in the bone defects was evaluated three-dimensionally. Results: Bone volume $(B V)$ and bone surface $(B S)$ in the tibiae of both the US and Control groups demonstrated the highest values 1 week after the operation, with no significant differences between the groups. At 2 weeks after the operation, the $B V$ and $B S$ values in both groups had decreased, but the decrease was smaller in the US group than the Control group. At 3 weeks after the operation, the $B V$ and $B S$ values in the Control group were significantly lower than those in the US group. Conclusion: LIPUS stimulation can prevent bone loss during the healing of bone defects.
\end{abstract}

This article is freely accessible online.

Correspondence to: Satoshi Yokose, DDS, Ph.D., Division of Endodontics and Operative Dentistry, Department of Restorative and Biomaterials Sciences, Meikai University School of Dentistry, Saitama, Japan. Tel/Fax: +81 492792736, e-mail: s-yokose@dent.meikai.ac.jp

Key Words: LIPUS, bone defect, 3D analysis, micro CT, rat tibia.
The healing of the extraction socket influences subsequent treatment in clinical situations such as severe alveolar bone resorption caused by periapical cysts, root fracture, or severe periodontal disease. Shortening the bone healing period while preserving bone quantity would be of benefit to patients as well as clinicians. Therefore, accelerating bone healing is clinically important.

It is well known that the application of mechanical stress stimulates bone metabolism. This is supported by Wolff's law, which states that bone can adapt to the load under which it is placed and that mechanical stress promotes bone metabolism (1). Recent studies have demonstrated that bone metabolism can be controlled by mechanical stress, which stimulates not only bone resorption but also bone formation (2). From a clinical point of view, it is more important to investigate the mechanism of how mechanical stress affects bone metabolism. However, the detailed mechanism remains unclear.

Considerable attention has focused on the application of low-power ultrasonic pulses (low-intensity pulsed ultrasound: LIPUS) as a non-invasive treatment approach (3). LIPUS is used in a variety of clinical applications, such as bone fracture treatment, treatment of periodontal disease, peri-implantitis, and ultrasonic irrigation in endodontics (4). For these reasons, we used LIPUS to analyze the effect of mechanical stress on bone metabolism.

Micro-focus computed tomography (micro CT) is very useful for investigating the fine structure of bone, and some studies have used micro CT imaging to analyze bone structure in the XY, YZ, and YZ section planes. However, $\mathrm{CT}$ generates only two-dimensional images. In order to analyze the precise structure of bone tissue, threedimensional (3D) images must be constructed using micro CT imaging data. 


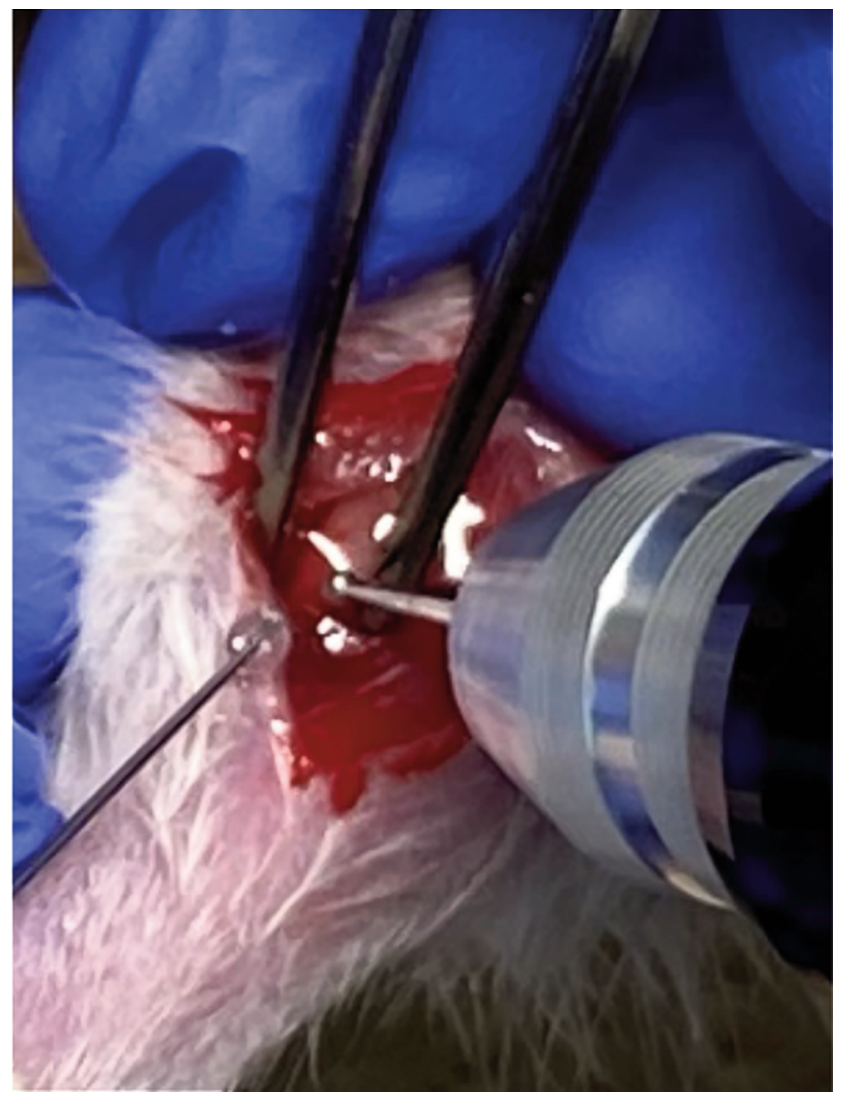

Figure 1. A bone defect was created in each tibia at the level of approximately $2 \mathrm{~mm}$ above the ankle using a dental steel bur ( $\phi 1 \mathrm{~mm})$ at high speed under a spray of normal saline.

In this study, $\mathrm{CT}$ images of rat tibia bone defects and the tibia treated with LIPUS were compared in terms of volume of new trabecular bone formation using 3D morphological assessment. The bone regenerative effect of LIPUS was evaluated using micro CT 3D morphological assessment of rat tibiae bone defects to characterize the effect of LIPUS on bone metabolism.

\section{Materials and Methods}

Preparation of bone defect models. The study was approved by the Animal Experiment Committee of Meikai University (No B2101). Sixteen 10-week-old female Sprague-Dawley (SD) rats were used in this study. The rats were housed in an experimental animal room $\left(20-26^{\circ} \mathrm{C}, 40-60 \%\right.$ humidity, and 12 -h light/dark cycle) and fed a standard laboratory diet and water. After anesthetization with isoflurane $(3.0 \%, 1.0 \mathrm{l} / \mathrm{min})$, skin incisions were made at both the right and left tibia. A bone defect was created in each tibia at a level of approximately $2 \mathrm{~mm}$ above the ankle using a dental steel bur $(\phi 1 \mathrm{~mm})$ at high speed under a spray of normal saline (Figure 1). The bone defect was made in the trabecular bone (Figure 2).

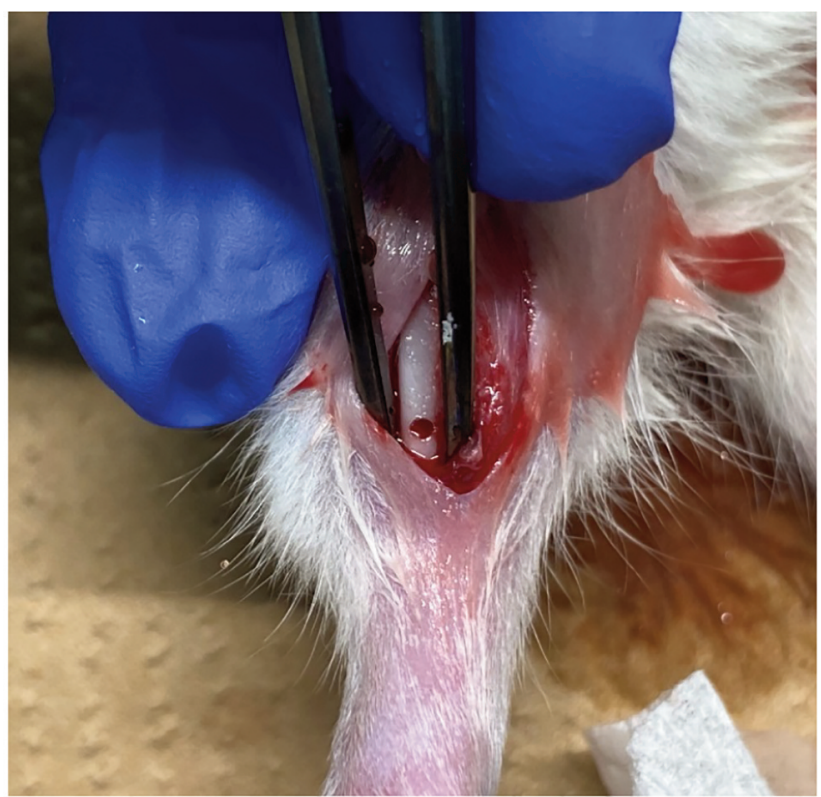

Figure 2. A 1.0-mm bone defect in rat tibia.

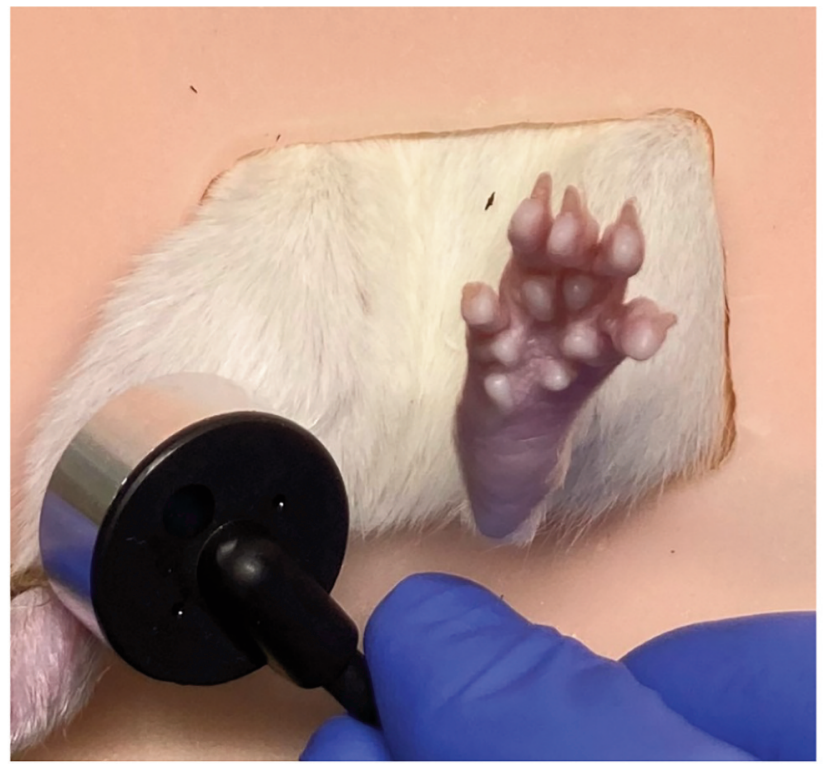

Figure 3. Low-intensity pulsed ultrasound was applied after placing the animal in a box that restricted excessive movements.

LIPUS exposure. A LIPUS exposure system (OSTEOTRONV, Saitama, Japan) was used in this study according to the manufacturer's instructions. The LIPUS exposure apparatus was set to deliver a $750-\mathrm{kHz}$ repeating pulsation at $1.0 \mathrm{kHz}$, with a spatial intensity of $60 \mathrm{~mW} / \mathrm{cm}^{2}$. Rats were exposed to LIPUS for 20 min daily. LIPUS was performed after placing the animal in a box that restricted excessive movement (Figure 3 ). The right tibia 


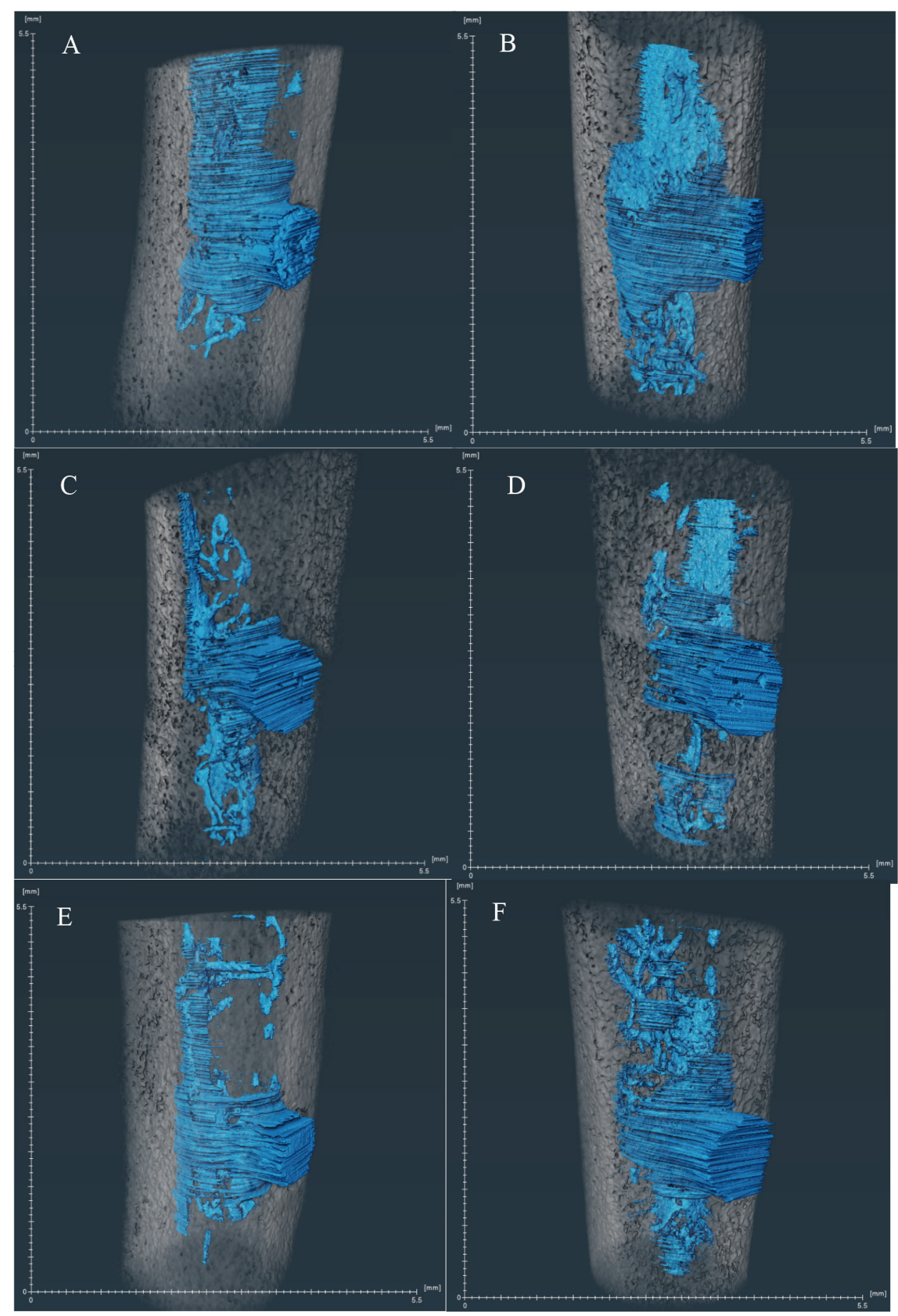

Figure 4. 3D models of newly formed bone tissue as revealed by blue color in the tibia of Control group ( $A, C$, and $E)$ and US group $(B, D$, and $F)$ rats at 1 week ( $A$ and $B), 2$ weeks ( $C$ and $D$ ), and 3 weeks ( $E$ and $F$ ) after the operation.

was treated with ultrasound, and ultrasound gel was applied directly on the tibia before LIPUS (US group), and the opposite side of the tibia received gel without ultrasound treatment (Control group). The right tibia was treated with LIPUS daily for 3 weeks.
Micro CT analysis. Rats were sacrificed at 1,2, and 3 weeks, and micro CT imaging was performed using a Cosmo Scan FX (Rigaku, Tokyo, Japan) with the following parameters: tube voltage $90 \mathrm{kV}$, tube current $88 \mu \mathrm{A}$, exposure time $2 \mathrm{~min}$, resolution $10 \mu \mathrm{m}$ isotropic. Micro CT images were evaluated in three dimensions (XY, YZ, and XZ sections). 
A region of interest (ROI) for each tibia was obtained by drawing new trabecular bone formation at the injured regions. The ROI was traced in each dimension (XY, YZ, and YZ sections) semiautomatically as described below $(\mathrm{a}, \mathrm{b})$, and $3 \mathrm{D}$ images were reconstructed using Thermo Scientific Amira ${ }^{\mathrm{TM}}$ software (Thermo Fisher Scientific, Waltham, MA, USA):

a. Evaluation of images in XY sections: the ROI was traced using the Lasso and Brush tool in Amira software, and the pixel value was set within fixed limits. The ROI was traced after a line connecting the edges of damaged cortical bone was drawn.

b. Revision of images in $\mathrm{YZ}$ and $\mathrm{XZ}$ sections: the portions of the images (a) that deviated as described were modified in the $\mathrm{YZ}$ and $\mathrm{XZ}$ sections. By tracing the ROI in the $\mathrm{XY}, \mathrm{YZ}$, and $\mathrm{XZ}$ sections of micro CT images, 3D models (3DMs) were reconstructed using Amira software. Microstructural indices [bone volume (BV) and bone surface (BS)] were calculated directly from the 3DMs.

Histologic analysis. To examine the effect of LIPUS treatment on fracture healing of the rat tibia at the tissue and/or cellular levels, we used three rats per group for the histological evaluation. The animals were anesthetized with an overdose of isoflurane and euthanized at 1,2, and 3 weeks. Defect sites were removed along with surrounding bone and fixed in $10 \%$ formalin at $4^{\circ} \mathrm{C}$. The specimens were then decalcified with KCX (FALMA, Tokyo, Japan) at $4^{\circ} \mathrm{C}$. Each tibia specimen was embedded in paraffin and serially sectioned using a microtome. Sections were stained with hematoxylin-eosin (H-E) for histological analysis.

Statistical analysis. The Wilcoxon rank sum test was used for statistical analyses. Results are reported as the mean \pm standard deviation. A level of significance of $5 \%$ was adopted for all tests $(p<0.05)$.

\section{Results}

Figure 4 shows 3DMs of new bone formation in the defect regions of rat tibia at 1 week (Figure $4 \mathrm{~A}$ and B), 2 weeks (Figure 4C and D), and 3 weeks (Figure 4E and F) after the operation. Newly formed bone structure, as indicated by blue color, can be observed in the Control (Figure 4A, C, and E) and US (Figure 4B, D, and F) groups. Newly formed bone that expanded from the defect region in cortical bone to the bone marrow spaces can be observed in the tibia specimens of both the Control and US groups.

Figure 5 shows a time course of the changes in BV (Figure 5A) and BS (Figure 5B) values for newly formed bone tissue in the tibia from 3DMs in the Control and US groups. Both the BV and BS values were highest at 1 week after the operation and eventually decreased until 3 weeks after the operation. Although there were no significant differences between the BV and BS values at 1 and 2 weeks after the operation, the values in the US group were higher than in the Control group at 3 weeks.

Figure 6 shows the results of histological examinations of the tibia at 1 (Figure 6A and $\mathrm{B}$ ) and 3 (Figure 6C and D) weeks after the operation. At 1 week after the operation, the defect region in the cortical bone was occupied by

\section{A}

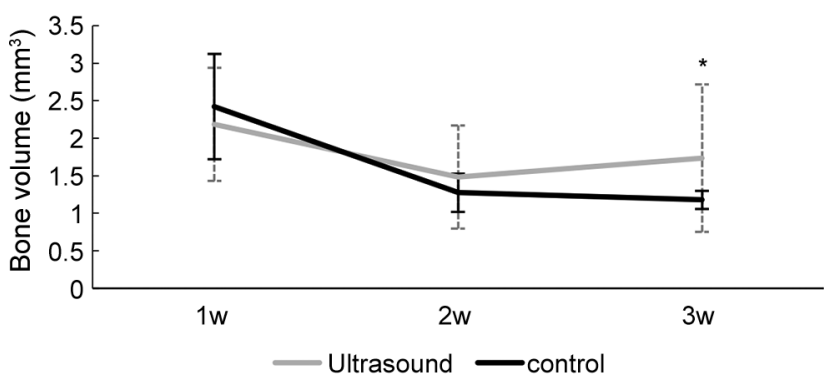

B

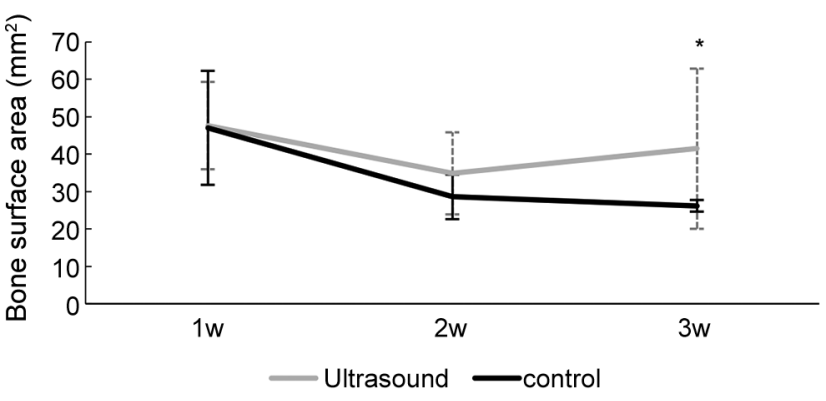

Figure 5. Time course of changes in bone volume (A) and bone surface area $(B)$ as analyzed with $3 D$ models. Data are presented as the mean $\pm S D$ of 5 or 6 rats in each group. * Significant difference from the Control group at $p<0.05$ as determined using the Wilcoxon rank sum test.

newly formed trabecular bone that expanded into the bone marrow spaces. There were no remarkable histological changes between the Control (Figure 6A) and US (Figure $6 \mathrm{~B})$ groups. At 3 weeks after the operation, the defect region in the cortical bone was almost completely repaired, and the amount of trabecular bone in the marrow spaces had decreased. There were no differences in histological features between the Control (Figure 6C) and US (Figure 6D) groups.

\section{Discussion}

Micro CT, which has been widely used to examine the fine structure of bone tissues, is indispensable for analyses of the 3D structure of bone tissues. In the present study, we used micro CT along with 3DMs to examine the effect of LIPUS on bone metabolism during the healing process in rat tibia with an introduced defect. H-E staining of sections prepared from the bone tissues was used to analyze the process histologically, but it is difficult to accurately judge bone formation and bone resorption via $\mathrm{H}-\mathrm{E}$ staining due to difference in the sites from which the sections are prepared. Indeed, we found no histological differences in the tibia between the Control and US groups; however, the 3DMs 


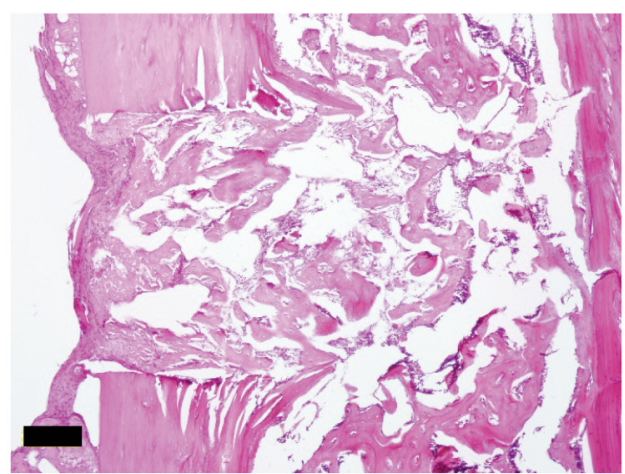

A: Control at 1 week

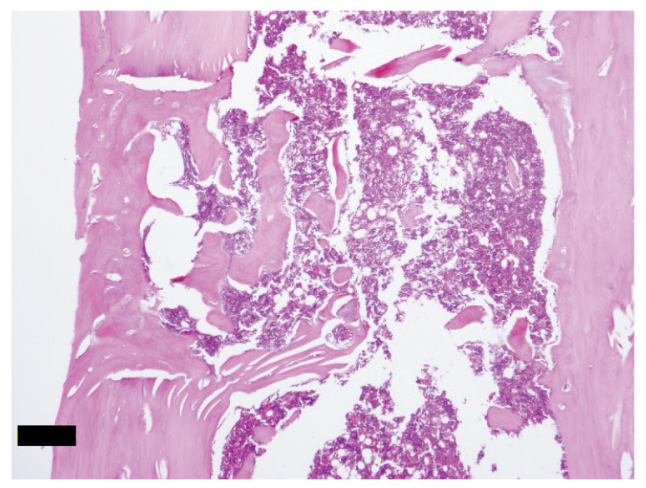

C: Control at 3 weeks

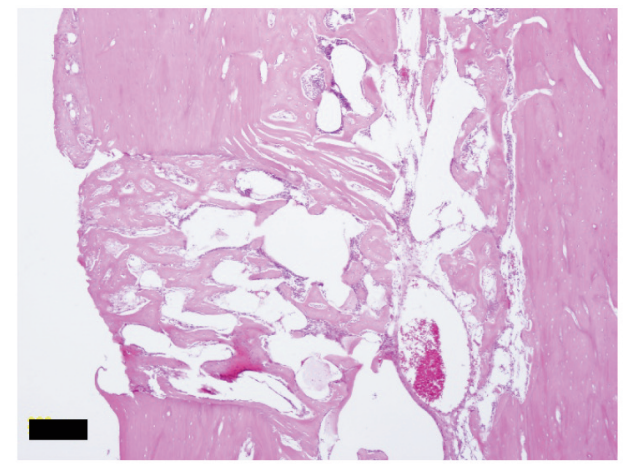

B: US at 1 week

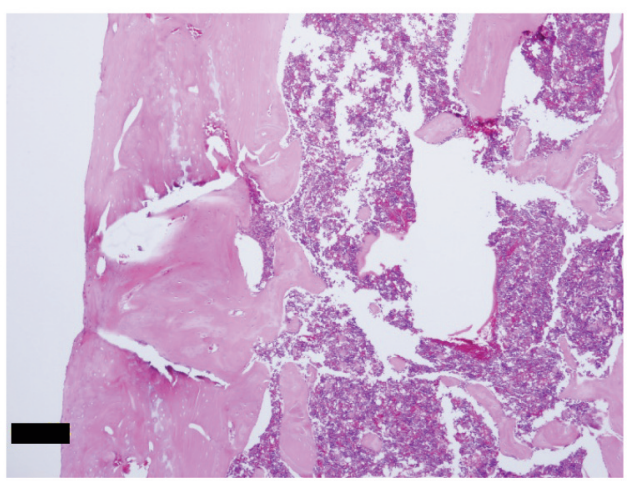

D: US at 3 weeks

Figure 6. Hematoxylin and eosin staining of sections of tibia of the Control (A and C) and US (B and D) groups at 1 week (A and B) and 3 weeks (C and D) after the operation. Scale bar: $200 \mu \mathrm{m}$.

revealed that LIPUS affected bone metabolism during the bone healing process. These results indicate that although LIPUS does not promote bone formation, it does prevent bone loss and maintains the volume of bone formed in defects in the tibia.

The fracture healing process is divided into an inflammatory phase, cell growth phase, callus formation phase, and remodeling phase. The callus formation phase usually occurs 1 to 4 weeks after bone fracture $(5,6)$. Several in vivo experimental studies (7-9) indicated that LIPUS has no effect on the remodeling phase of fracture healing but instead affects the earlier inflammatory or callus formation phases of healing. Azuma et al. (10) reported more extensive bone bridging at the fracture site in bones continuously treated with LIPUS for 17-24 days after operation. Additionally, they showed significant increases in the stiffness and maximum torque of LIPUS-treated bone compared with untreated bone. Konno et al. (11) reported that initiating LIPUS stimulation in the early period following bone injury-during the inflammatory and cell growth phases, before callus formation - effectively accelerates bone union. These studies support our results, as we confirmed that early use of LIPUS is effective for bone fracture treatment and strengthens the bone.

Changes observed in tissues and cells after LIPUS treatment may also be associated with acoustic streaming and cavitation $(12,13)$. Rawool et al. (14) reported that LIPUS treatment affects angiogenesis in fracture healing. They found that LIPUS treatment for 10 days resulted in a $33 \%$ increase in vascularity at the osteotomy site of dog ulna. The authors suggested that LIPUS induces mechanical vibrations that increase the permeability of the cell membrane to calcium ions, leading to increased micromechanical fluid pressure of blood flow.

Sasaki et al. (15) reported accelerated capillary vessel formation in LIPUS-stimulated bone. Based on our results, we hypothesize that LIPUS induces angiogenesis that affects bone metabolism in defect areas of the tibia. However, we did not examine angiogenesis in our study; thus, further investigations are needed to elucidate the mechanism.

In conclusion, using 3D morphological evaluation of micro CT images, we found that continuous LIPUS treatment for 3 
weeks prevents bone loss during the healing process in rat tibia with an introduced defect.

\section{Conflicts of Interest}

All Authors state that they have no conflicts of interest in relation to this study.

\section{Authors' Contributions}

Study design and conduct: KW and SY; Data collection: KW, MK, KK, and YH; Data analysis: KW, MK, RS, HK, and YH; Data interpretation: KW, MK, YH, and SY; Drafting the manuscript: KW and SY; Revising the manuscript: HS, HK and SY; Approval of the final manuscript: KW, MK, YH, and SY. SY; Corresponding author.

\section{Acknowledgements}

This study was supported in part by a Grant-in-Aid for Scientific Research (16K20449) from the Ministry of Education, Science, and Culture of Japan.

\section{References}

1 Wolff J: Das gesetz der transformation der knochen. A Hirshwald 1: 1-152, 1892.

2 Robling AG and Turner $\mathrm{CH}$ : Mechanical signaling for bone modeling and remodeling. Crit Rev Eukaryot Gene Expr 19(4): 319-338, 2009. PMID: 19817708. DOI: 10.1615/ critreveukargeneexpr.v19.i4.50

3 Claes L and Willie B: The enhancement of bone regeneration by ultrasound. Prog Biophys Mol Biol 93(1-3): 384-398, 2007. PMID: 16934857. DOI: 10.1016/j.pbiomolbio.2006.07.021

4 Karumuri SK, Rastogi T, Beeraka K, Penumatcha MR and Olepu SR: Ultrasound: A revenant therapeutic modality in dentistry. J Clin Diagn Res 10(7): ZE08-ZE12, 2016. PMID: 27630969. DOI: $10.7860 / \mathrm{JCDR} / 2016 / 18584.8190$

5 Suda R, Ozawa H and Takahashi H (eds): Bone Biology, $2^{\text {nd }}$ ed. Tokyo, Ishiyaku pub, 2016.

6 Matsumoto Dental University Graduate School of Hard Tissue Research Group: Hard Tissue Research Handbook. Nagano, Matsumoto Dental University Press, 2008.
7 Pilla AA, Mont MA, Nasser PR, Khan SA, Figueiredo M, Kaufman JJ and Siffert RS: Non-invasive low-intensity pulsed ultrasound accelerates bone healing in the rabbit. J Orthop Trauma 4(3): 246-253, 1990. PMID: 2231120. DOI: 10.1097/ 00005131-199004030-00002

8 Wang SJ, Lewallen DG, Bolander ME, Chao EY, Ilstrup DM and Greenleaf JF: Low intensity ultrasound treatment increases strength in a rat femoral fracture model. J Orthop Res 12(1): 4047, 1994. PMID: 8113941. DOI: 10.1002/jor.1100120106

9 Hantes ME, Mavrodontidis AN, Zalavras CG, Karantanas AH, Karachalios $\mathrm{T}$ and Malizos KN: Low-intensity transosseous ultrasound accelerates osteotomy healing in a sheep fracture model. J Bone Joint Surg Am 86(10): 2275-2282, 2004. PMID: 15466739. DOI: 10.2106/00004623-200410000-00021

10 Azuma Y, Ito M, Harada Y, Takagi H, Ohta T and Jingushi S: Low-intensity pulsed ultrasound accelerates rat femoral fracture healing by acting on the various cellular reactions in the fracture callus. J Bone Miner Res 16(4): 671-680, 2001. PMID: 11315994. DOI: 10.1359/jbmr.2001.16.4.671

11 Konno M, Asano H, Fujii Y, Konishi Y and Muragaki Y: Effects of low-intensity pulsed ultrasound on callus formation: A comparative morphological study. J Tokyo Wom Med Univ 87(4): 108-116, 2017. DOI: 10.24488/jtwmu.87.4_108

12 Hill C: Ultrasonic exposure thresholds for changes in cells and tissues. The Journal of the Acoustical Society of America 50(1A): 90-90, 2016. DOI: 10.1121/1.1977776

13 Dyson M: Non-thermal cellular effects of ultrasound. Br J Cancer Suppl 5: 165-171, 1982. PMID: 6950755.

14 Rawool NM, Goldberg BB, Forsberg F, Winder AA and Hume E: Power Doppler assessment of vascular changes during fracture treatment with low-intensity ultrasound. J Ultrasound Med 22(2): 145-153, 2003. PMID: 12562119. DOI: 10.7863/ jum.2003.22.2.145

15 Sasaki H, Monden K, Yoshinari M and Yajima Y: Comparison of angiogenesis in bone defect healing process due to the difference in the frequency of low-intensity pulsed ultrasound (LIPUS). Journal of Hard Tissue Biology 25(2): 157-164, 2017. DOI: $10.2485 /$ jhtb.25.157

Received December 2, 2021 Revised January 2, 2022 Accepted January 3, 2022 